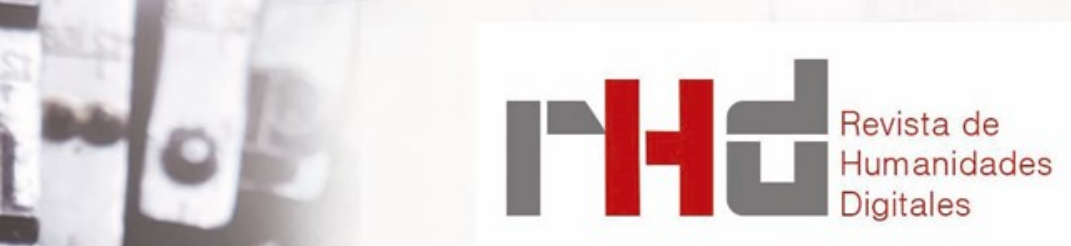

\title{
Comparación de los sentimientos en The Puppet Masters y las emociones en Twitter durante la pandemia COVID-19: un análisis cuantitativo del miedo al contagio
}

Dirección

Clara Martínez Cantón

Gimena del Rio Riande

Francisco Barrón

\section{Secretaría}

Romina De León
Comparison of Feelings in The Puppet Masters and Emotions on Twitter during the COVID-19

Pandemic: a quantitative analysis of fear of contagion
José Luis ARROYO-BARRIGÜETE

Universidad Pontificia Comillas

¡larroyo@comillas.edu

https://orcid.org/0000-0002-3660-3933
Lucía BARCOS REDÍN

Universidad Pontificia Comillas

lbarcos@comillas.edu

https://orcid.org/0000-0002-7843-3653

\section{RESUMEN}

Empleando técnicas computacionales de text mining, en este trabajo se compara la respuesta emocional a nivel social detectada en la investigación de Dubey (2020) durante los primeros meses de la pandemia, con la que aparece en una de las obras icónicas en literatura de ciencia ficción: The Puppet Masters (1951), de Robert A. Heinlein. Esta novela describe un escenario que, en lo esencial, es muy similar a la actual crisis global sanitaria causada por la COVID-19. Los resultados demuestran que existe una enorme similitud entre ambos patrones emocionales. El hecho de que una novela de hace casi setenta años exhiba un patrón emocional extraordinariamente similar al que hemos observado en la sociedad durante la pandemia, pone de manifiesto no solo la capacidad de Heinlein para dotar de verosimilitud a sus obras, sino las similitudes entre las ansiedades que rodearon la Guerra Fría y las ansiedades ligadas a las primeras etapas de la epidemia.

\section{PALABRAS CLAVE}

Heinlein, The Puppet Masters, Twitter, análisis de sentimientos, pandemia.

\begin{abstract}
Using text mining techniques, we compare the emotional response at a social level detected by Dubey's research (2020) during the pandemic, with the one that appears in one of the iconic novels in science fiction literature: The Puppet Masters (1951), by Robert A. Heinlein. This novel describes a scenario that, in essence, is very similar to the current global health crisis caused by COVID-19. The results show that there is an enormous similarity between both emotional patterns. The fact that a novel from almost seventy years ago exhibits an emotional pattern extraordinarily similar to the one observed in society during the pandemic, shows not only Heinlein's ability to give his works verisimilitude, but similarities between anxieties surrounding the Cold War and anxieties surrounding the early stages of the pandemic.
\end{abstract}

\section{KEYWORDS}

Heinlein, The Puppet Masters, Twitter, Sentiment Analysis, Pandemics. 


\section{INTRODUCCIÓN}

Durante una pandemia como la causada por la COVID-19, los seres humanos pueden manifestar diversas reacciones psicológicas negativas ligadas a la ansiedad y el miedo, pero también positivas, de confianza y esperanza. Ciertamente, el campo de la psicología de las emociones es extraordinariamente complejo, hasta el punto de que no existe una única teoría aceptada de forma universal sobre las emociones humanas básicas. Sin embargo, sí existe consenso sobre la idea de que una simple dicotomía entre sentimientos positivos o negativos no resulta adecuada, aunque, más allá de eso, encontramos diferentes marcos conceptuales. Ekman (1992), por ejemplo, identifica seis emociones básicas: alegría (joy), asco (disgust), ira (anger), miedo (fear), sorpresa (surprise) y tristeza (sadness), mientras que Plutchik (2009) las amplía a ocho, añadiendo confianza (trust) y anticipación (anticipation). De acuerdo con la teoría de Plutchik, la combinación de dos emociones primarias genera díadas, de modo que, por ejemplo, la mezcla de alegría y anticipación genera optimismo (optimism). Es precisamente este último marco conceptual sobre el que se basa Dubey (2020) para analizar los tweets relacionados con la COVID-19 en 12 países, entre el 11 y el 31 de marzo de 2020, todo ellos escritos en inglés. En dicho trabajo, se recogieron y analizaron en torno a 50.000 tweets de cada país cada 4 días, concluyendo que la mayoría de las personas en los países analizados adoptaron un enfoque positivo: aunque los sentimientos de miedo y tristeza están muy presentes, la emoción predominante es confianza. La herramienta de análisis fue el lexicón de emociones NRC, el mismo que se emplea en este trabajo y que se explicará más adelante. La tabla 1 muestra el análisis detallado por país, con el peso relativo de cada una de las ocho emociones básicas de Plutchik, así como el promedio global simple (no ponderado por población) de los doce países.

\begin{tabular}{|c|c|c|c|c|c|c|c|c|}
\cline { 2 - 8 } \multicolumn{1}{c|}{} & Alegría & $\begin{array}{c}\text { Anticipa- } \\
\text { ción }\end{array}$ & Asco & $\begin{array}{c}\text { Confian- } \\
\text { za }\end{array}$ & Ira & $\begin{array}{c}\text { Mie- } \\
\text { do }\end{array}$ & $\begin{array}{c}\text { Sorpre- } \\
\text { sa }\end{array}$ & Tristeza \\
\hline Australia & 7.4 & 16.7 & 6.2 & 21.8 & 9.2 & 19.3 & 5.7 & 13.7 \\
\hline Bélgica & 8.7 & 17.5 & 5.3 & 25.9 & 9.9 & 15.6 & 9.8 & 7.3 \\
\hline China & 6.2 & 17.3 & 4.4 & 24.4 & 10.7 & 21.4 & 5.6 & 10.2 \\
\hline Francia & 4.3 & 10.0 & 5.2 & 15.4 & 18.2 & 22.4 & 5.8 & 18.6 \\
\hline Alemania & 6.8 & 19.9 & 4.2 & 21.7 & 7.9 & 20.2 & 7.2 & 12.1 \\
\hline India & 12.5 & 16.6 & 4.6 & 21.2 & 9.7 & 18.7 & 6.5 & 10.2 \\
\hline Italia & 11.3 & 16.2 & 4.6 & 22.5 & 7.3 & 17.8 & 8.2 & 12.2 \\
\hline Holanda & 7.7 & 13.3 & 5.1 & 18.9 & 7.3 & 23.0 & 4.9 & 19.7 \\
\hline España & 9.7 & 16.0 & 5.1 & 21.7 & 9.0 & 18.7 & 7.1 & 12.9 \\
\hline Suiza & 6.4 & 10.0 & 4.7 & 15.4 & 6.3 & 28.1 & 4.7 & 24.5 \\
\hline Reino Unido & 11.1 & 17.0 & 5.1 & 21.9 & 8.5 & 17.2 & 7.3 & 11.9 \\
\hline EE. UU. & 10.7 & 9.3 & 6.7 & 19.4 & 15.9 & 17.2 & 8.6 & 12.3 \\
\hline Promedio & 8.6 & 15.0 & 5.1 & 20.8 & 10.0 & 20.0 & 6.8 & 13.8 \\
\hline
\end{tabular}

Tabla 1. Peso (en porcentaje) de cada una de las emociones en los tweets de cada país. Fuente: adaptado de Dubey (2020). 
Ciertamente, en términos medios, la confianza es la emoción predominante, levemente por encima del miedo (figura 1), seguidas de la anticipación y la tristeza. Se trata de un patrón interesante, pues en el periodo considerado se produjo un incremento exponencial tanto en contagiados como en fallecidos, algo que sin duda fue motivo de alarma. Según datos del Johns Hopkins Coronavirus Resource Center ${ }^{1}$, el 11 de marzo de 2020 el número de infectados era de 126.559 y el de fallecidos de 4.611 , mientras que el 31 de ese mismo mes los contagiados ascendían a 872.076 y los fallecidos a 44.629. Es decir, los infectados se habían multiplicado casi por siete y los fallecidos casi por diez. Incluso en este escenario, tal y como el análisis de Dubey (2020) pone de manifiesto, en términos generales, las emociones que se transmitían a través de Twitter en los 12 países analizados eran razonablemente positivas, dado el contexto.

25

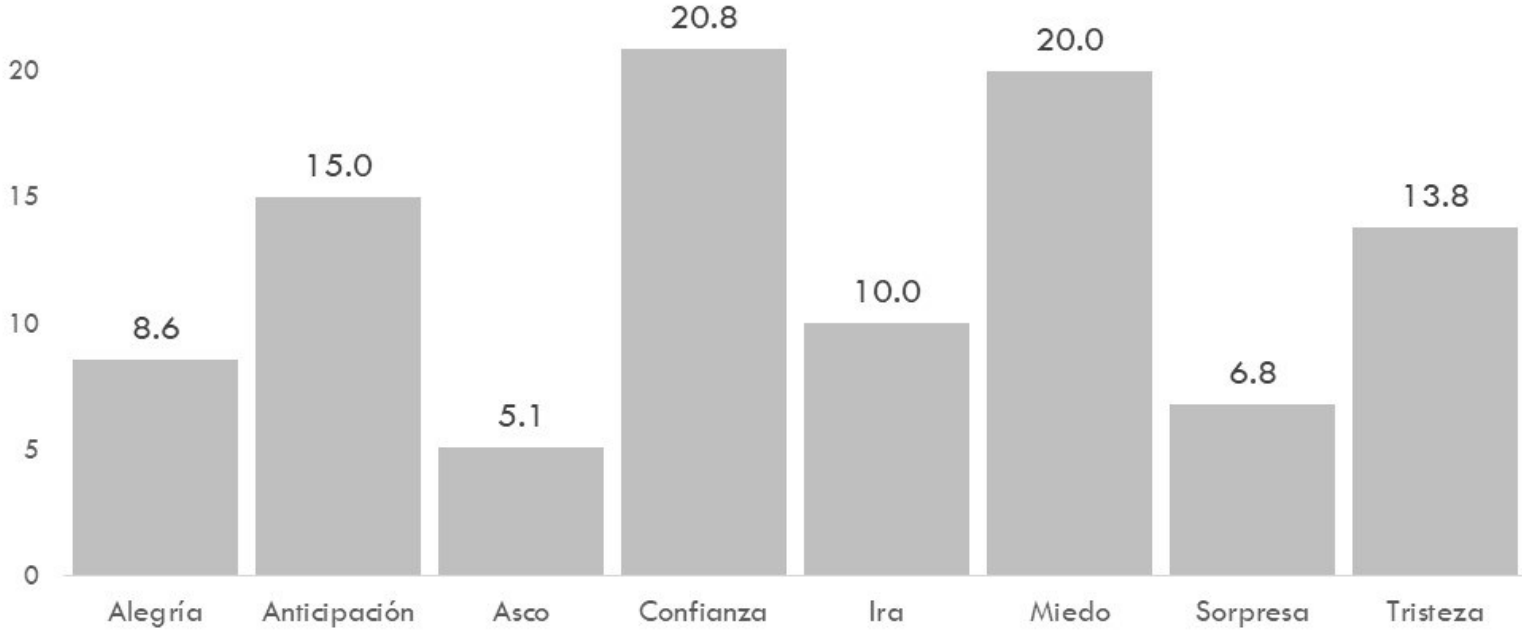

Figura 1. Peso promedio de cada una de las emociones en los tweets de los doce países. Fuente: elaboración propia a partir de Dubey (2020).

A efectos de evaluar la robustez del resultado de Dubey, es decir, de determinar hasta qué punto estamos ante un reflejo real del comportamiento humano durante las primeras etapas de la pandemia, se ha decidido contrastar sus resultados con los obtenidos por otro grupo de investigadores, que realizan un análisis completamente diferente (Aslam et al., 2020). Dichos autores estudiaron las emociones de 141.208 titulares de noticias sobre la COVID-19, publicados en las 25 fuentes de noticias en inglés de mayor impacto (Reuters, BBC, Yahoo News, South China Morning Post, National Post, Daily Mail UK, CNBC, The Guardian, CNN, etc.) entre el 15 de enero de 2020 y el 3 de junio de 2020, y seleccionando aquellos que incluían la palabra coronavirus. La herramienta de análisis en este caso también es el lexicón NRC y el periodo analizado es similar, correspondiéndose con las primeras etapas de la pandemia, aunque, como puede observarse, los datos proceden de una fuente completamente diferente. Esto permite precisamente hacer una comparación de los resultados y verificar si, con independencia de los datos estudiados, tweets por una parte y titulares de noticias por el otro, el patrón emocional es similar. Como se observa en la figura

\footnotetext{
1 Accesible desde: https://coronavirus.jhu.edu/map.html.
} 
2, ciertamente el patrón emocional es prácticamente idéntico, siendo la diferencia más destacada el hecho de que en el caso de los titulares de prensa, el miedo supera a la confianza en peso relativo. De hecho, existen varios trabajos que han reportado resultados muy similares o prácticamente idénticos, como es el caso de Kumar et al. (2020), que analizaron 10.000 tweets del 17 al 30 de marzo de 2020, o Kausar et al. (2021) sobre un conjunto de 1.305.000 tweets entre el 21 de junio y el 20 de julio de 2020. Esto nos lleva a la conclusión de que, efectivamente, el patrón emocional detectado por Dubey (2020) es realmente un reflejo del comportamiento humano observable durante las primeras etapas de la pandemia.

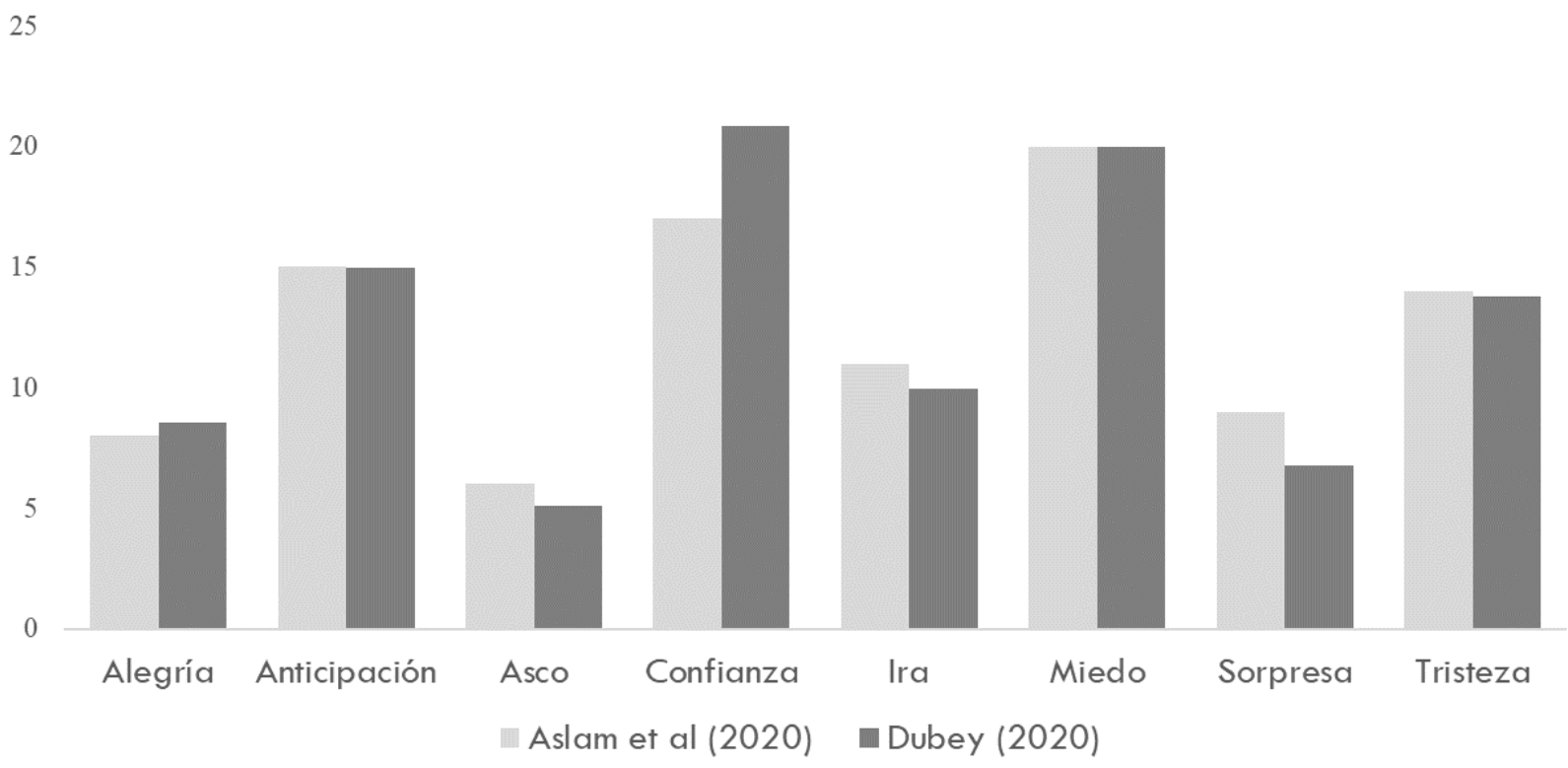

Figura 2. Comparación de los resultados Aslam et al. y Dubey. Fuente: elaboración propia a partir de Aslam et al. (2020) y Dubey (2020).

Por tanto, hemos identificado el patrón de sentimientos durante uno de los momentos críticos de la pandemia y, la pregunta que nos hacemos es hasta qué punto la literatura de ciencia ficción plantea esquemas similares, focalizando dicha pregunta en una obra icónica de este género. Concretamente se ha elegido The Puppet Masters (Heinlein, 2000), escrita por Robert A. Heinlein ${ }^{2}$ y adaptada al cine en el año 1994. Esta novela describe la historia de una invasión por parte de parásitos alienígenas, y el hilo narrativo de la obra se articula en torno al miedo al contagio y la paranoia imperante de quiénes estarán o no infectados, pudiendo contagiar a otros. De hecho, esta paranoia llega al extremo de terminar con tabúes sociales tan profundamente arraigados, y más en el año en que se escribió la novela, como el desnudo: dado que los parásitos son una especie de babosas que se adhieren al cuerpo, se impone el desnudo en todos los ámbitos de la vida social, a fin de detectar a los infectados. Cualquier persona vestida es considerada automáticamente sospechosa. La novela se inicia con el primer contagio en una pequeña localidad de lowa que progresivamente se extiende a lo largo de todo el país y, como se descubre más adelante,

\footnotetext{
2 Heinlein está considerado uno de los mejores escritores de ciencia ficción del siglo XX. Fue ganador de cuatro premios Hugo y nombrado Gran Maestro Damon Knight Memorial por la SFWA (Science Fiction and Fantasy Writers of America) en 1974.
} 
por todo el planeta. El protagonista, miembro de una agencia de seguridad nacional denominada The Section, trata de impedir el avance de la invasión, lo que finalmente consigue, aunque solo en EE. UU., pues cuando la novela termina, no queda claro si otros países, incluida la URSS, han sido capaces de lograrlo.

Como señala MacDermott (1982), The Puppet Masters es "a classic Cold War 'paranoia' tale dealing in body-snatching by aliens" (p. 268) y, si bien se ha criticado esta obra por diversas razones, la más frecuente es precisamente una posible lectura palimpséstica demasiado obvia y maniquea, ligada al Macartismo y al miedo imperante en la sociedad norteamericana a la existencia de un comunismo anidado en su propio seno. Como señala Lockett (2007), la novela "establishes a clear contiguity between the aliens and communism, leaving no room for a more ambivalent or nuanced interpretation of the allegory" (p. 46). Ciertamente, el trabajo de Heinlein, y The Puppet Masters no es una excepción, sino que es a la vez producto y partícipe de la Guerra Fría (Brown, 2008). Heinlein está simplemente expresando en su obra el miedo al contagio comunista, recogiendo así las ansiedades propias de la Guerra Fría. La pregunta de investigación que se plantea en este trabajo está ligada precisamente a este hecho. Pretendemos averiguar hasta qué punto Heinlein recogió en su obra un patrón emocional similar al detectado durante las etapas iniciales de la pandemia $y$, en función de los resultados, responder a dos cuestiones íntimamente relacionadas: hasta qué punto el autor fue capaz de recoger en su obra un patrón emocional realista, y en qué medida dicho patrón, ligado a las ansiedades de la Guerra Fría, es similar al detectado durante la pandemia. De encontrarse similitudes sustanciales, se confirmaría no solo la habilidad de Heinlein, sino que además mostraría cómo el miedo al contagio, independientemente de que fuera físico como en la pandemia, o ideológico como en la Guerra Fría, conduce a un patrón emocional semejante.

Por último, es preciso indicar que The Puppet Masters presenta dos características que la hacen especialmente adecuadas para llevar a cabo el análisis mencionado: estar centrada en la batalla contra la invasión/infección, e incidir en una atmósfera de paranoia ligada al miedo al contagio: diversas obras icónicas de la literatura de ciencia ficción narran los acontecimientos posteriores a la pandemia, con una visión postapocalíptica, por lo que no es posible establecer el paralelismo claro con la situación actual, y otras no inciden de forma tan importante en el miedo al contagio; miedo que sí ha caracterizado a la presente y a pasadas pandemias.

\section{MATERIAL Y MÉTODOS}

El análisis de la novela se ha llevado a cabo íntegramente en R (R Core Team, 2013), un entorno de programación orientado al análisis estadístico, empleando diversas librerías para elaborar el código: tidytext (Silge \& Robinson, 2016), ggplot2 (Wickham, 2016), fmsb (Nakazawa, 2018), tidyr (Wickham \& Henry, 2019), scales (Wickham, 2018), stringr (Wickham, 2019), dplyr (Wickham et al., 2020), y readtext (Benoit \& Obeng, 2020). El preprocesamiento de los datos implica ciertas transformaciones sobre las 96.849 palabras que componen la novela, transformación 
de letras mayúsculas a minúsculas, supresión de signos de puntuación, eliminación de las denominadas stopwords o palabras vacías (palabras que no tienen un significado en sí mismas, sino que modifican o acompañan a otras, tales como artículos, pronombres o preposiciones) y por supuesto la tokenización (división del texto en piezas más pequeñas o tokens, en nuestro caso, palabras). Esta limpieza resulta imprescindible como paso previo al análisis cuantitativo. Posteriormente, se ha empleado el lexicón de emociones NRC (Mohammad \& Turney, 2013b), un listado de 14.182 unigramas (palabras) en lengua inglesa y sus asociaciones con las ocho emociones básicas de Plutchik. Así, por ejemplo, la palabra abandoned está asociada a sentimientos de tristeza, miedo e ira. Esta asociación se ha llevado a cabo mediante un proyecto de crowdsourcing, cuyos detalles pueden consultarse en Mohammad y Turney (2013a). De este modo, cada token (palabra) se ha vinculado a las emociones correspondientes según el lexicón, dando lugar a un listado de las ocho emociones de Plutchik con sus respectivas frecuencias absolutas, es decir, el número de veces que dicha emoción aparece en el texto. El análisis se ha realizado por palabras, no considerando frases $u$ otras estructuras superiores. Dividiendo cada una de estas frecuencias entre la suma de todas ellas, obtenemos la frecuencia relativa o peso de la emoción correspondiente, lo que es un indicador de su relevancia en el conjunto de la obra.

No obstante, hemos de realizar una precisión metodológica que, de hecho, constituye una debilidad, tanto del análisis que se lleva a cabo en este artículo, como del trabajo de Dubey (2020). En ambos casos se ha trabajado con el lexicón de emociones NRC, dado que se trata de un recurso ya utilizado y testado por numerosos investigadores desde su publicación. Es decir, muchos años de uso por diferentes académicos avalan su fiabilidad. No obstante, se trata de una herramienta que presenta una obvia limitación: las palabras pueden asociarse con diferentes intensidades (o grados) de una emoción. Como los propios autores del lexicón señalan "most people will agree that the word condemn is associated with a greater degree of anger (or more anger) than the word irritate" (Mohammad \& Turney, 2020). Precisamente, para solventar esta limitación, estos autores han publicado recientemente el NRC Emotion Intensity lexicón (NRC-EIL), que además de llevar a cabo la asociación entre palabras y las ocho emociones básicas, incluye puntuaciones de intensidad. Sin embargo, la primera versión de este nuevo lexicón se publicó en marzo de 2020, por lo que aún no ha sido suficientemente testada. Adicionalmente, si queremos asegurar la comparabilidad de los resultados con los obtenidos por Dubey (2020), lo más adecuado es seguir su misma metodología, lo que implica emplear el lexicón NRC.

\section{RESULTADOS Y DISCUSIÓN}

Tras analizar los sentimientos presentes en The Puppet Masters, encontramos que la emoción predominante es confianza (19.7\%), seguida de miedo (16.9\%), anticipación (13.6\%), tristeza (1 1.9\%) e ira (1 1.6\%). Las otras tres emociones, asco, alegría y sorpresa tienen pesos por debajo del $10 \%$, del $9.1 \%, 9.1 \%$ y $8.0 \%$ respectivamente. Si comparamos este resultado con los obtenidos por Dubey (2020) para el promedio de los doce países analizados, observamos que las dife- 
rencias son relativamente pequeñas (tabla 2). Únicamente en los sentimientos de asco y miedo encontramos diferencias superiores a dos puntos porcentuales. Y, de hecho, la mayor diferencia, en el sentimiento de asco, parece plenamente justificada por la naturaleza de los parásitos descritos en la obra de Heinlein: una especie de "gray slug" (Heinlein, 2000, p. 34), que el autor describe como "disgustingly ugly, but not more so than slime in a pond-not as much so as maggots in garbage" ( $p$. 80). Es decir, que se debe a un elemento coyuntural de la novela (la forma escogida por Heinlein para representar a los parásitos), y no a un aspecto clave en el esquema emocional de la obra.

\begin{tabular}{|r|c|c|c|c|c|c|c|c|}
\cline { 2 - 8 } \multicolumn{1}{c|}{} & Alegría & $\begin{array}{c}\text { Anticipa- } \\
\text { ción }\end{array}$ & Asco & Confianza & Ira & Miedo & Sorpresa & Tristeza \\
\hline $\begin{array}{r}\text { Twitter } \\
\text { (Dubey, } \\
\text { 2020) }\end{array}$ & 8.6 & 15.0 & 5.1 & 20.8 & 10.0 & 20.0 & 6.8 & 13.8 \\
\hline $\begin{array}{r}\text { The Puppet } \\
\text { Masters }\end{array}$ & 9.1 & 13.6 & 9.1 & 19.7 & 11.6 & 16.9 & 8.0 & 11.9 \\
\hline Diferencia & -0.6 & 1.4 & -4.0 & 1.1 & -1.7 & 3.0 & -1.2 & 1.9 \\
\hline
\end{tabular}

Tabla 2. Peso (en porcentaje) de las emociones en Twitter y en The Puppet Masters, así como la diferencia entre ambas. Fuente: elaboración propia.

De hecho, si representamos gráficamente la tabla (figura 3), resulta sorprendente la considerable similitud entre ambos patrones emocionales. En ambos casos las cinco emociones principales son confianza, miedo, anticipación, tristeza e ira, precisamente en ese orden, y con pesos relativamente parecidos.

- The Puppet Masters $\square$ Dubey (2020)

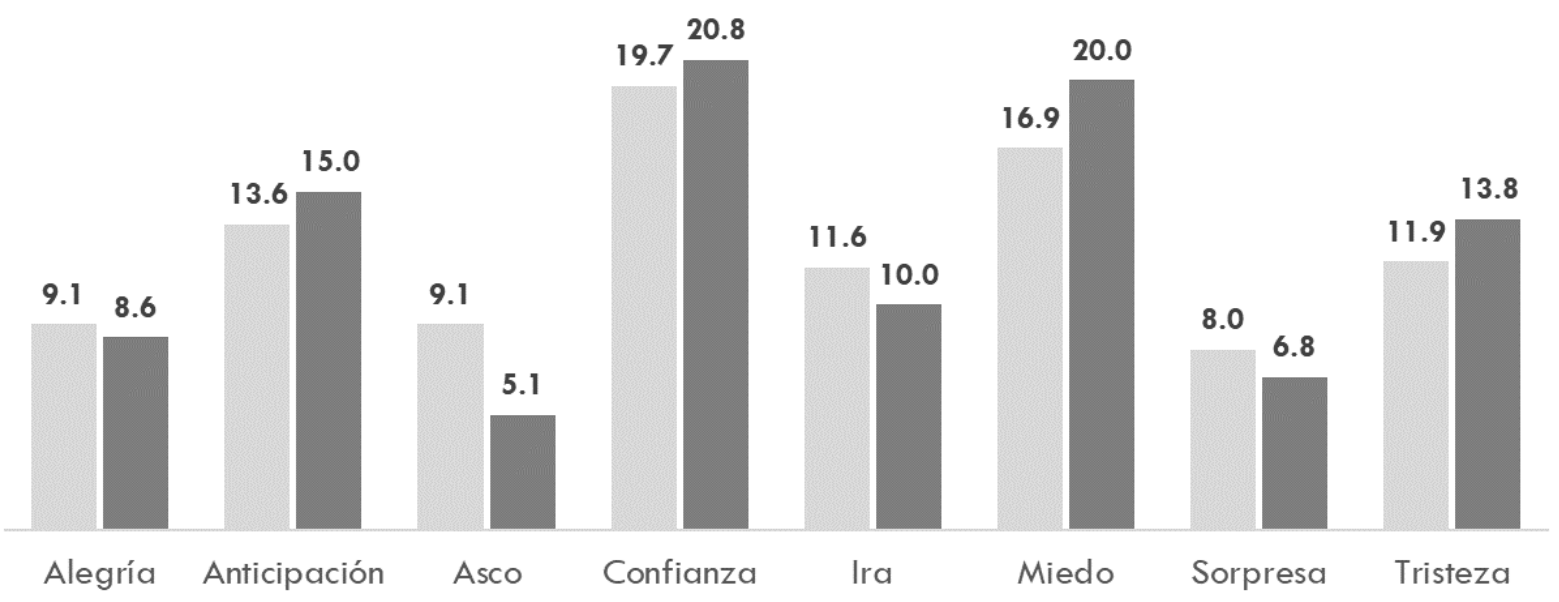

Figura 3. Comparación de patrones entre The Puppet Masters y lo sentimientos identificados en Twitter por Dubey (2020). Fuente: elaboración propia.

No obstante, esto podría deberse a que, con cierta frecuencia, encontramos que un mismo autor emplea patrones similares en todas sus obras. Es decir, podría darse el caso de que el patrón de emociones encontrado fuese característico en toda la obra de Heinlein, y no fruto de un esfuerzo deliberado por su parte para representar la realidad durante una pandemia/invasión. Esto sucede, por ejemplo, con H. P. Lovecraft, escritor cuyos cuentos y novelas presentan una consi- 
derable homogeneidad en lo que al patrón emocional se refiere, independientemente de la temática de la obra (Arroyo-Barrigüete, 2021 a y 2021 b) ${ }^{3}$. Para descartar este particular, se ha llevado a cabo el mismo análisis con otra de sus obras icónicas, Stranger in a Strange Land (Heinlein, 1987), ganadora del Premio Hugo a la mejor novela, y cuya temática es completamente diferente a la de The Puppet Masters. En la figura 4 se muestra la comparación de ambas obras y, como puede comprobarse, encontramos una estructura de emociones marcadamente diferente. Es decir, no resulta posible achacar el patrón detectado en The Puppet Masters al estilo de Heinlein, sino que parece relacionado con el contenido de la obra. $O$, expresado de otro modo, podemos concluir que dicho patrón es el fruto de un esfuerzo intencionado por parte del autor para reflejar la atmósfera propia de una situación de pandemia.

\section{Whe Puppet Masters $\quad$ Stranger in a Strange Land}

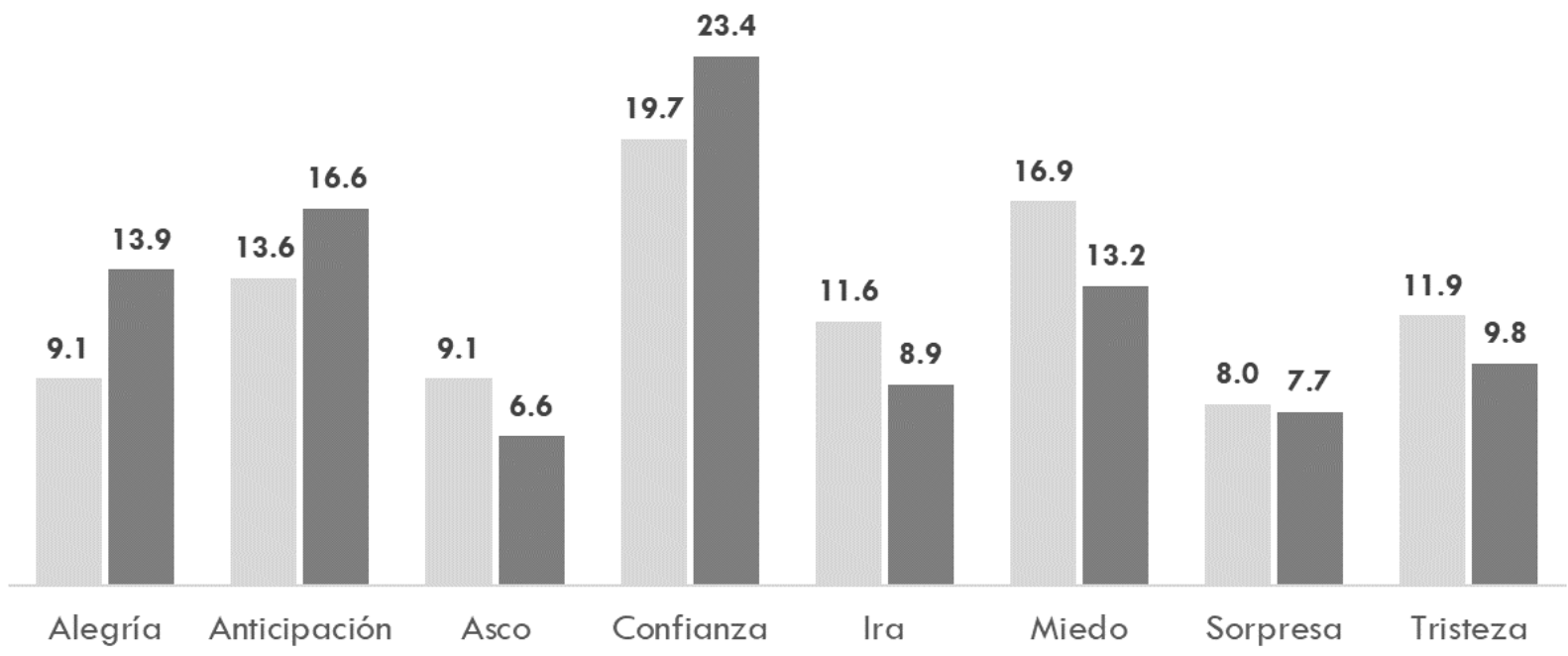

Figura 4. Comparación de patrones entre The Puppet Masters y Stranger in a Strange Land. Fuente: elaboración propia.

Descartado, por lo tanto, que la coincidencia de patrones se deba a un sesgo estilístico de Heinlein, podemos extraer varias conclusiones. En primer lugar, parece que el escritor fue capaz de reproducir en su obra un esquema emocional bastante realista. Esto incide en una interesante línea de investigación en el campo de la lingǘstica computacional, pues pone de manifiesto la plausibilidad de usar novelas para estudiar los patrones emocionales en épocas pasadas. Actualmente, las redes sociales, medios electrónicos y páginas web, en combinación con las técnicas modernas de análisis de datos, permiten estudiar emociones a nivel social. Sin embargo, esto no resulta factible cuando se trata de periodos anteriores. El resultado obtenido apunta a la factibilidad de llevar a cabo este análisis a través de obras realistas escritas en el periodo de interés.

En relación con la segunda cuestión mencionada en la introducción, la obra fue escrita en 1951, es decir hace casi setenta años y, sin embargo, el patrón es el mismo que se ha observado

\footnotetext{
${ }^{3}$ Concretamente, tal y como señala Arroyo-Barrigüete (2021 a), Lovecraft emplea un patrón prácticamente idéntico en varias de sus obras icónicas. Y ampliando el estudio a una gran parte de su producción literaria, 54 obras, Arroyo-Barrigüete (2021 b) concluye que, la práctica totalidad (50 de ellas) se agrupan en dos únicos patrones emocionales, que, adicionalmente, son relativamente similares entre sí.
} 
durante la pandemia de la COVID-19, lo que nos lleva a pensar, que las respuestas emocionales ante una situación de este tipo no parecen haber cambiado en exceso. Es más, el patrón emocional de miedo al contagio físico que se da en la pandemia resulta extraordinariamente parecido al miedo al contagio ideológico que se daría en la Guerra Fría. De algún modo, la respuesta sociológica parece ser en ambos casos prácticamente idéntica. Sin embargo, en relación con este particular, se observa una enorme paradoja, las semejanzas son mucho mayores si consideramos el patrón global medio de los 12 países, tal y como se hace en la figura 3, que si consideramos únicamente a EE. UU. (figura 5). Como apunta Patterson (2010), "the story of Robert A. Heinlein is the story of America in the twentieth century" (p. 12), por lo que cabría esperar justo lo contrario. $Y$, sin embargo, vemos una clara anomalía en el sentimiento de ira, inesperadamente alto, siendo de hecho el tercero en cuanto a peso relativo. Escapa a los objetivos del presente trabajo analizar las razones de dicha anomalía, que también aparece en Francia, aunque quizá podamos aventurarnos a pensar que está relacionado con el ambiente de crispación política que se vivía en EE. UU. en el periodo considerado. En resumen, encontramos importantes similitudes entre el patrón de sentimientos en The Puppet Masters y los observados por Dubey (2020) en el conjunto de los doce países analizados, si bien, paradójicamente, las similitudes son menores cuando consideramos únicamente EE. UU. (figura 6).

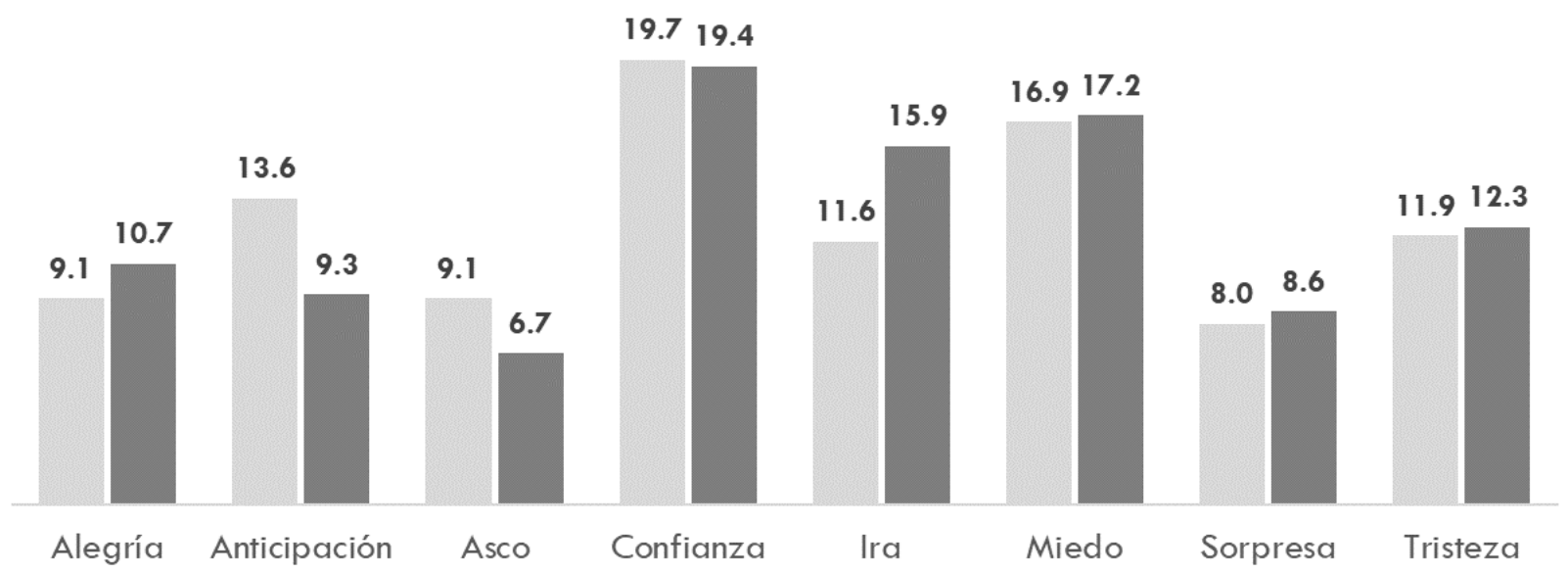

Figura 5. Comparación de patrones entre The Puppet Masters y lo sentimientos identificados en Twitter por Dubey (2020) en EE. UU. Fuente: elaboración propia. 


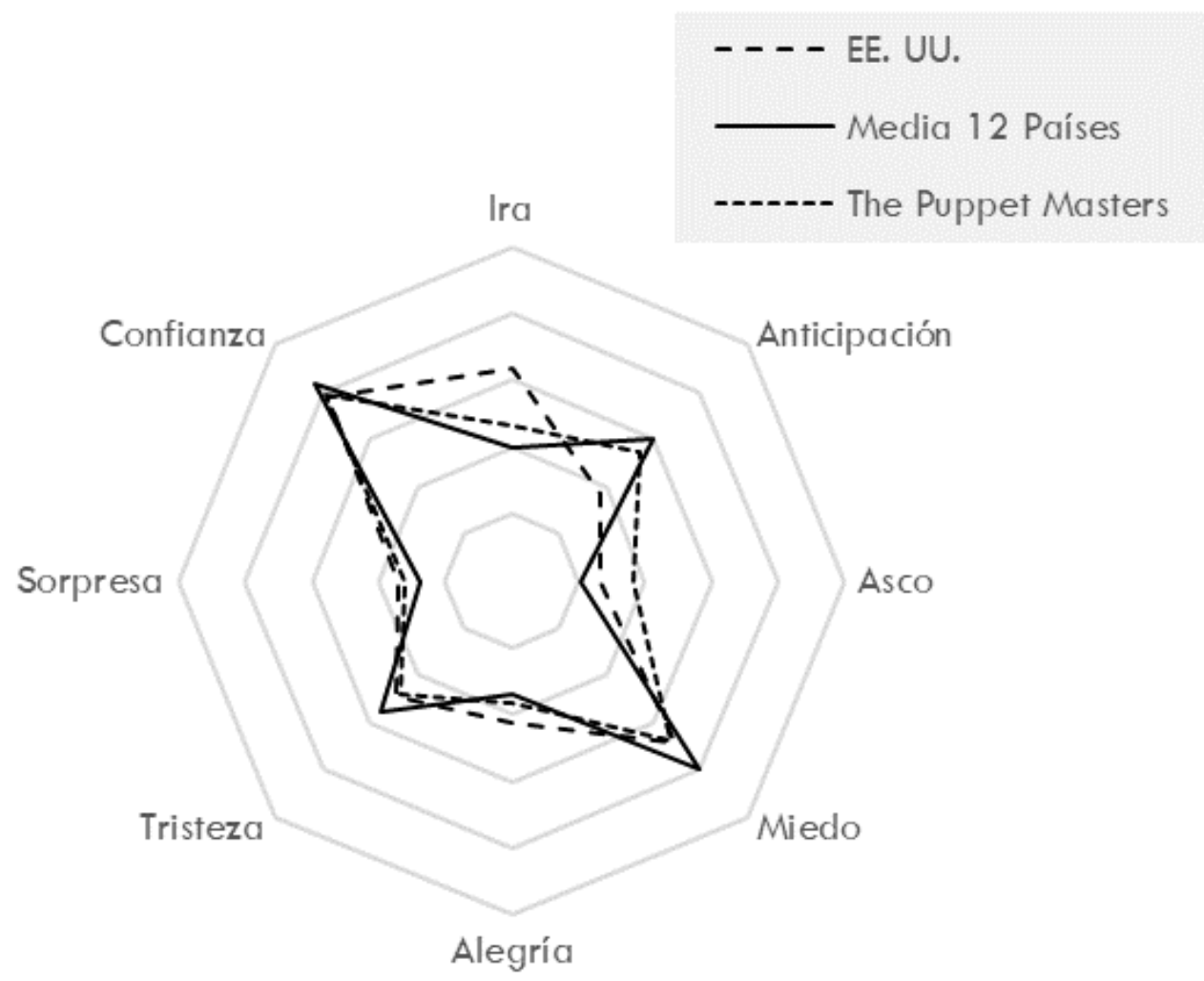

Figura 6. Comparación de patrones entre The Puppet Masters y lo sentimientos identificados en Twitter por Dubey (2020) en EE. UU. y en el promedio de los 12 países analizados. Fuente: elaboración propia.

\section{CONCLUSIONES}

En este trabajo se ha comparado el patrón emocional observado durante la pandemia y más concretamente durante el mes de marzo de 2020, con el que aparece en una de las obras icónicas de ciencia ficción, The Puppet Masters de Robert A. Heinlein. Dubey (2020), analizando los mensajes enviados a través de Twitter por usuarios de 12 países en el período mencionado, llega a la conclusión de que "[the] majority of the people throughout the world are taking a positive and hopeful approach, there are instances of fear, sadness and disgust exhibited worldwide" (p. 1). Concretamente, si consideramos el valor promedio de la muestra, comprobamos que la emoción dominante es confianza (peso del 20.8\%), seguida del miedo $(20.0 \%)$, anticipación (15.0\%), tristeza (13.8\%) e ira (10.0\%). Tras llevar a cabo un análisis similar sobre la novela The Puppet Masters, comprobamos que existen importantes similitudes, pues los sentimientos más relevantes no solo son los mismos, sino que aparecen exactamente en el mismo orden: confianza (19.7\%), miedo (16.9\%), anticipación (13.6\%), tristeza (11.9\%) e ira (11.6\%). Tras descartar que esta coincidencia pueda deberse a un sesgo estilístico del autor, hemos de concluir que es fruto de un esfuerzo intencionado para recrear una ambientación verosímil. Es decir, Heinlein planteó en The Puppet Masters un patrón emocional muy concreto, que, aparentemente, buscaba recoger la atmósfera propia de la batalla contra una pandemia que amenazaba con aniquilar a la raza humana tal y como la conocemos. En realidad, Heinlein estaba reflejando los miedos y ansiedades de un contexto cultural muy 
específico, el propio de la sociedad norteamericana durante la Guerra Fría, ligada al Macartismo y al miedo a la existencia de un comunismo arraigado en su propio seno. Es decir, recoge el miedo al contagio ideológico, percibido como una amenaza a la sociedad y los valores norteamericanos. Hoy, casi setenta años después de la publicación de esa obra y, enfrentados a una pandemia real, observamos que el patrón emocional que emerge es sorprendentemente parecido al descrito por Heinlein. Parece por tanto que, a nivel social, la respuesta emocional es muy similar en ambas situaciones. Esto nos lleva a dos conclusiones diferentes: pone de manifiesto la destreza narrativa de Heinlein; y apunta a que el miedo al contagio, con independencia de su naturaleza, parece generar una respuesta emocional similar y bastante estable en el tiempo.

\section{AGRADECIMIENTOS}

Los autores agradecen a los/las evaluadores/as anónimos/as sus comentarios y sugerencias, que sin duda han contribuido a mejorar sustancialmente la versión final del manuscrito.

\section{REFERENCIAS BIBLIOGRÁFICAS}

Aslam, F., Awan, T. M., Syed, J. H., Kashif, A., \& Parveen, M. (2020). Sentiments and Emotions Evoked by News Headlines of Coronavirus Disease (COVID-19) Outbreak. Humanities and Social Sciences Communications, 7(1), 1-9.

Arroyo-Barrigüete, J. L. (2021a). El estilo narrativo en H.P. Lovecraft: un análisis cuantitativo de The Call of Cthulhu y otras obras icónicas. Cultura, Lenguaje y Representación, XXV, 47-63. http://dx.doi.org/10.6035/clr.2021.25.3

Arroyo-Barrigüete, J. L. (2021 b). Stylistic Study of Lovecraft's Fiction Writings: Sentiment Analysis Based on Plutchik's Wheel of Emotions. Working Paper (Enero, 2021). https://cutt.ly/ $\underline{\text { bnnINeB }}$

Benoit, K., \& Obeng, A. (2017). Readtext: Import and Handling for Plain and Formatted Text Files (Versión 0.76) [Software]. https://CRAN.R-project.org/package=readtext

Brown, J. F. (2008). Heinlein and the Cold War: Epistemology and Politics in the Puppet Masters and Double Star. Extrapolation, 49(1), 109-121.

Dubey, A. D. (2020). Twitter Sentiment Analysis during COVID19 Outbreak. https:// papers.ssrn.com/sol3/papers.cfm?abstract id $=3572023$

Ekman, P. (1992). Are there Basic Emotions? Psychological Review, 99(3), 550-553.

Heinlein, R. A. (1987 [1961]). Stranger in a Strange Land. Ace.

Heinlein, R. A. (2000 [1951]). The Puppet Masters. Rosetta Books.

Kausar, M. A., Soosaimanickam, A., \& Nasar, M. (2021) Public Sentiment Analysis on Twitter Data during COVID-19 Outbreak. International Journal of Advanced Computer Science and Applications (IJACSA), 12(2). http://dx.doi.org/10.14569/IJACSA.2021.0120252

Kumar, A., Khan, S. U., \& Kalra, A. (2020). COVID-19 Pandemic: A Sentiment Analysis: A Short Re- 
view of the Emotional Effects Produced by Social Media Posts during this Global Crisis. European Heart Journal, 41 (39), 3782-3783.

Lockett, C. (2007). Domesticity as Redemption in "The Puppet Masters": Robert A. Heinlein's Model for Consensus. Science Fiction Studies, 34(1), 42-58.

MacDermott, K. A. (1982). Ideology and Narrative: The Cold War and Robert Heinlein. Extrapolation, 23(3), 254-269.

Mohammad, S., \& Turney, P. (2013a). Crowdsourcing a Word-Emotion Association Lexicon. Computational Intelligence, 29(3), 436-465.

Mohammad, S., \& Turney, P. (2013b). NRC Emotion Lexicon (NRC Technical Report). http:// saifmohammad.com/WebPages/NRC-Emotion-Lexicon.htm

Mohammad, S., \& Turney, P. (2020). The NRC Emotion Intensity Lexicon (NRC-EIL Technical Report). https://saifmohammad.com/WebPages/AffectIntensity.htm

Nakazawa, M. (2018). fmsb: Functions for Medical Statistics Book with some Demographic Data (Versión 0.6.3) [Software]. https://CRAN.R-project.org/package=fmsb

Patterson Jr, W. H. (2010). Robert A. Heinlein: In Dialogue with His Century, Volume 1: Learning Curve (1907-1948). Tor.

Plutchik, R. (2009). Emotions: A General Psychoevolutionary Theory. En Klaus R. Scherer, \& P. Ekman (Eds.), Approaches to Emotion (pp. 197-219). Psychology Press.

R Core Team. (2013). R: A Language and Environment for Statistical Computing [Software]. http:// www.R-project.org/

Silge, J., \& Robinson, D. (2016). Tidytext: Text Mining and Analysis Using Tidy Data Principles in R. Journal of Open Source Software, 1(3), 37.

Wickham, H. (2016). ggplot2: Elegant Graphics for Data Analysis. Springer-Verlag.

Wickham, H. (2018). scales: Scale Functions for Visualization (Versión 1.0.0) [Software]. https:// CRAN.R-project.org/package $=$ scales

Wickham, H. (2019). Stringr: Simple, Consistent Wrappers for Common String Operations (Versión 1.4.0) [Software]. https://CRAN.R-project.org/package=stringr

Wickham, H., \& Henry, L. (2019). tidyr: Tidy Messy Data (Versión 1.0.0) [Software]. https://CRAN.R -project.org/package=tidyr

Wickham, H., François, R., Henry, L., \& Müller, K. (2020). dplyr: A Grammar of Data Manipulation (Versión 0.8.5) [Software]. https://CRAN.R-project.org/package $=$ dplyr 